\title{
Algunas consideraciones sobre investigaciones y docencia
}

\author{
Roberto Garduño Vera ${ }^{1}$
}

\section{RESUMEN}

Se anotan diver sos as pec tos so bre la na tu ra le za y orien ta ción de la in ves ti ga ciónque se de sa rro lla en la UNAM en las áreas de cien cia y tec no lo gía y hu ma ni da des. Tam bién se se ña lan al gu nas ta reas pro pias del in ves ti gador y su in ci den cia en el pro ce so de en se ñan za-aprendizaje, desarrollado en nuestra Máxima Casa de Estudios.

En particular se sugierendirectrices de cómo el investigador en el área bibliotecológica puede lograr una mayor participación con el Cole gio de Biblio te colo gía de la Fa cul tad de Filo so fía y Le tras, en lo que res pec ta a vin cu lar en for ma más es tre cha a la in ves ti ga ción en bibliote cologíaque se de sarrolla en elCen tro Uni versitario de In vestigaciones B iblio te co ló gi cas (CUIB), con las cáte dras que ofre ce el Colegio mencionado.

Se se ña la que se ría de sea ble for ma li zar un con ve nio de tu to rías en tre el Co le gio de Bi blio te co lo gía y el CUIB, con el fin de que es tudian tes de los úl ti mos se mes tres de la li cen cia tu ra o maes tría en esta área, tra ba ja ran en for ma di rec ta con el in ves ti ga dor para de sa rrollar su trabajo de tesis. Esto permitiría entre otros aspectos reforzar el programa de formación de investigadores de este Centro.

Tam bién se des ta ca en el do cu men to la al ter na ti va de con si de rar que elCen tro, bajo con ve nio, ofre cie ra cur sos no su je tos a la cu rrícu la del Colegio, pero sí con valor en créditos. Esos cursos serían basados en las líneas de investigación del CUIB.

\section{ABSTRACT}

Di ver se as pects about the na tu re and orien ta tion of re search that is be ing produced in the UNAM in the areas of scien ce, te chno logy and hu ma ni ties. Cer tain appro pria te tasks for the re sear cher are sugges ted as well as their re le vance for the tea ching-learning process that is carried out in our National University.

More pre ci sely, cer tain di rec ti ves are sugges ted as to how the li brary scien ce re sear cher can ob tain grea ter par ti ci pa tion with the Co legio de Biblio te co logíain theFacul tad de Fi lo sofía y Le tras re garding clo se relations bet ween the li brary scien ce re search ca rried ourt in the Centro Universitario de Investigaciones Bibliotecológicas (CUIB) and the classes offered in the aforementioned college.

It is poin ted our thath it would be use ful to for ma li ze a tu to rial agre e ment bet ween theCo le gio de Bi blio te co lo gía and the CUIB, so that students in the last se mes ters of their ba che lor or mas ters pro gram could work di rec tly with the re sear cher. This would per mit, among other benefits, the strengthening of the program of the training of researcher of this Center.

This do cu ment also highlights the pos si bi lity that the Cen ter con si der, un der a bi la te ral agre e ment, offe ring cour ses that are not in the cu rri cu lum of the co lle ge, but that would be gi ven aca de mic cre dit. The se cour ses would be ba sed upon the re search foun da tions of the CUIB.

Investigar en un sentidogenéricosignificabuscarparadescribir y esto en un am bien te uni ver si ta rio el tér mi no ad quie re ma yor rigor. In ves ti garen la Uni ver si dad es bus car y crear co no ci mien to, bus car res pues ta a un fe nó me no dado. Esto con base en mé to dos de investigación rigurosos.

En di ver sos me dios de co mu ni ca ción so cial se ha se ña la do que la investigación en la UNAM requie re de es cla recercon preci sión su significado; reorienarla, valorarla como uno de los quehace- res fundamentales de la Universidad y llevar a efecto su promoción en forma eficaz.

Esos pun tos de vis ta son im por tan tes de con si de rar ya que lo que se pre ten de es me jo rar y en su caso en cau sar el con jun to de va riables que conforman la infraestructura de la investigación en la Universidad. Esto con un solo fin; contribuir al desarrollo integral del país. 
El tema in ves ti ga ción y los pun tos de ri va dos de la mis ma es una de las preo cu pa cio nes prio ri ta rias a dis cu tir en el Con gre so Univer si ta rio. Se ten drán en cuen ta los pun tos de vis ta de los uni versitarios, y seguramente también se tomará como parámetro orientador al Programa para la Modernización Educativa 1989. 1994. Esto re sul ta ra zo na ble si se tie nen pre sen tes los fi nes que la Universidad persigue para con la sociedad, fines que conviene es tén vin cu la dos a un pro gra ma in te gral de de sa rrollo. Esto, considero, no incidirá en la libertad de investigación.

Las ta reas de in ves ti gar ad quie ren ma yor sen ti do cuan do a los logros de éstas se les relaciona con fenómenos sociales para solucio nar al gún pro ble ma dado o para me jo rar y fa ci li tar el ni vel de vida de esa sociedad.

Al vincular la investigación con la docencia se realiza una interac ción aca dé mi ca en tre in ves ti ga do res y es tu dian tes, de estoresultará, de parte de los investigadores, compartir los conocimientos e ideas derivadas de su investigación. Los estudian tes a su vez apro ve cha rán esos co no ci mien tos y re troa li mentarán con sus ideas a los investigadores, logrando con esto aprovecharconocimientos emanados de la investigación para la formación de cuadros profesionales.

Las labores de investigación y docencia se encuentran planteadas como fun ciones sus tan ti vas de la UNAM, en la Ley Or gá ni ca que la sus ten ta y tam bién en otras dis po si cio nes le ga les de me nor jerarquía.

ElEs tatu to delPersonal Aca démico, ${ }^{2}$ en su ar tícu lo se gun do, señala:

"Las funciones del personal académico de la Universidad son: impartir educación, bajo el principio de li ber tad de cá te dra y de in vestigación, parafor mar profe sionis tas, in vestiga dores, profesores universitarios y técnicos útiles a la sociedad, organizar y realizar investigaciones principalmente acerca de temas y problemas de interés nacional, y desarrollar actividades conducentes a ex ten der con la ma yor am pli tud po si ble los be ne fi cios de la cultura..."

El artículo 61 del mismo Estatuto remarca:

"El per so nal aca dé mi co de ca rre ra, de me dio tiem po y de tiem po com ple to, tie ne la obli ga ción de de sem pe ñar la bo res do cen tes y de investigación..."

Con base en los pre cep tos le ga les se po dría en ten der que in ves tigación y docencia son inseparables. Crear conocimiento sin trans mi tir lo se ría mu ti lar a la en se ñan za. En se ñar sin te ner aportaciones de nuevosconocimientos se ría re pro du cir el acer vo del saber sin incrementarlo.

In ves ti ga ción y docen cia re quie ren mu tua men te, la pri me ra para re ci bir la in fluen cia de los alum nos y la se gun da para en ri que cer- se de la reflexión y experiencia de quienes están dedicados a la creación de nuevos conocimientos.

Al pa re cer la idea de vin cu lar la in ves ti ga ción con la do cen cia se orienta a los cursos curriculares que se imparten en las facultades. Sin embargo, una serie de situaciones impiden en diversos ca sos que in ves ti ga do res de ins ti tu tos y cen tros cum plan con este rubro.

Por ejem plo, se dan ca sos en que no exis ten cál cu los dis po ni bles de bi do a que un solo maes tro es ti tu lar de di ver sas ma te rias, ocasionando con ello poca oportunidad para que los investigadores que aún noim par ten cur sos cu rricu la res pue dan in gresaraés tos.

Este asun to en tre otros, re pre sen tan una preo cu pa ción que me rece ser ana li za da en for ma per ma nen te con el pro pó si to de bus car mecanismos que permitan, de manera efectiva y cotidiana, la vinculación más estrecha de la investigación y la docencia.

Sin em bar go, opi no que la la bor do cen te de in ves ti ga do res debe ver se con la ma yor am pli tud y no re du cir se a las ho ras cla se frente al grupo, por lo que considero que otras formas de participación de investigadores que conduzcan a la vinculación investigación-docencia, serían:

Asesorar a instituciones o a personas

Dirigir tesis de diferentes niveles

Impartir en distintos niveles cursos de actualización

Apo yar en el di se ño o ac tua li za ción de pla nes y pro gra mas de estudio

Elaborar materiales de apoyo a la docencia

\section{ALGUNAS CONSIDERACIONES SOBRE INVESTIGA- CIÓN Y DOCENCIA EN EL CUIB}

Tomando como referencia los puntos señalados con anterioridad, se ac ce dió a los ar chi vos y a los in for mes del per so nal de inves ti ga ción. Se en con tró que de 1987 a 1989 se ha re gis tra do un incrementofa vorable en el renglón de in vesti ga ción y docencia.

Algunos imparten cursos en el Colegio de Bibliotecología y otros en la Escuela Nacional de Biblioteconomía y Archivonomía. También, y de manera ocasional, algunos investigadores colaboran en impartición de cursos de actualización relacionados con su proyecto de investigación.

Algunos investigadores tienen actividades relacionadas con la docencia tales como asesoría a escuelas y dirección de tesis; otros han im partidocursos en universidadesex tranjeras, re lacionados con su investigación.

2 UNAM. Legislación : Estatuto del Personal Académico. México: La UNAM. Oficina del Abogado General. Dirección General de Estudios de Legislación Universitaria, 1987, p. 193. 
Otros investigadores asesoran a diferentes instituciones vinculan do tam bién de esta ma ne ra los co no ci mien tos ad quiri dos en el proceso de su investigación.

\section{ALGUNAS SUGERENCIAS}

Convendría formalizar un convenio de tutorías entre el Colegio de Bi blio te co lo gía y el CUIB, con el pro pó si to de que es tu diantes de los úl ti mos se mes tres de la li cen cia tu ra o maes tría, tra bajaran de manera directa con el investigador, con el fin de desarrollar su trabajo de tesis.

El convenio tendría que contemplar una justificación con argumentos suficientes respecto a la necesidad de esa vinculación, para evi tar, con ello, una car ga do cen te fuer te al in ves ti ga dor que le impida cumplir de manera satisfactoria sus tareas sustantivas de investigación.

Uno de los re sul ta dos de ese con ve nio tu to rial se ría de sa rro llar el in te rés en la in ves ti ga ción de quien fue ra el tu to ra do, con el propósi to de refor zarel progra ma de for ma ción de in ves ti ga do res de esta dependencia.

Otra alternativa a considerar es que el Centro, bajo convenio, ofre cie ra la al ter na ti va de im par tir cur sos no su je tos a la cu rrícur la del Co le gio, pero sí con va lor a cré di tos. Esos cur sos se rían basados en las líneas de investigación del CUIB.

\section{CITAS BIBLIOGRÁFICAS}

(1)UNAM. Le gislación: Estatu to delPersonalAcadémico. Méxi co: La UNAM, Ofi ci na del Abo ga do Ge ne ral, Di rec ción Ge ne ral de Estudios de Legislación Universitaria, 1987.

\section{BIBLIOGRAFÍA CONSULTADA}

Programa para la Modernización Educativa 1989-1994. México : 1989. 202 p.

Ruigarcía Torres, Armando. “Es posible vincular docencia e investigación?”. EN: El Financiero, 18 de enero de 1990.

UNAM. Legislación: EstatutodelPersonalAcadémico. Méxi co : La UNAM, Ofi ci na del Abo ga do Ge ne ral. Di rec ción Ge ne ral de Estudios de Legislación Universitaria, 1987. 290 p.

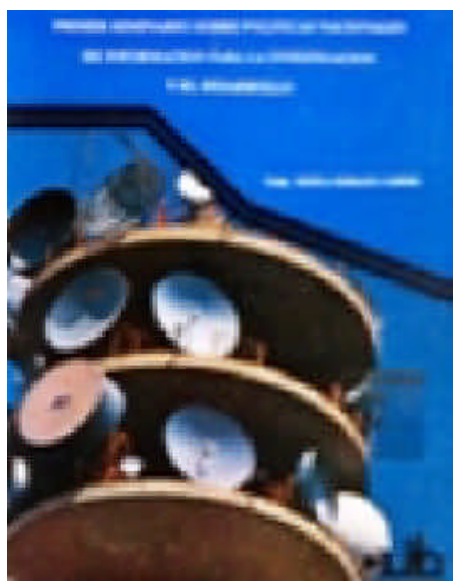

Primer se mi na rio sobre políticas nacionales de información para a investigación y el desarrollo

Morales, Estela 98 p. 